\title{
SCHRIFTKULTUR UND SCHWELLENKUNDE? ÜBERLEGUNGEN ZUM ZUSAMMENHANG VON LITERALITÄT UND LIMINALITÄT
}

\author{
ACHIM GEISENHANSLÜKE
}

La science se parle, la littérature s'écrit.

Roland Barthes

\section{Vor der Schwelle}

Eine Arbeitsgruppe, die sich mit dem Thema der Literalität und der Liminalität auseinandersetzt, sieht sich fast zwangsläufig mit verschiedenen Vorbehalten konfrontiert, die graduell voneinander differieren mögen, aber ein und dieselbe Sache betreffen. Das kann bei vorsichtigem Nachfragen zu dem eigentlichen Kern des Forschungsprojektes beginnen, und es kann mit Häme und Spott über ein Thema enden, das manchen an den Haaren herbeigezogen zu sein scheint. Wie auch immer die Reaktion derjenigen ausfällt, die sich zur Nachfrage aufgefordert fühlen: Sie reagieren auf eine Unbestimmtheit, die in der Sache selbst begründet liegt. Mit dem Thema der Literalität und der Liminalität stehen drei Dinge in Frage, die nicht einfach durch den Hinweis zu erledigen sind, dass Alliterationen schön klingen und der Arbeit des Literaturwissenschaftlers auch sonst gut anstehen. Wenn zugestanden wird, dass Begriffsklärung eine der grundlegenden Aufgaben der Wissenschaft ist, dann ergibt sich zum einen die Notwendigkeit, den meist relativ einsinnig gebrauchten Begriff der Literalität zu definieren, und zum anderen die, den metaphorisch-schillernden Begriff der Liminalität zu erläutern, um schließlich den Zusammenhang zwischen Literalität und Liminalität bestimmen zu können.

Was in diesem Zusammenhang allerdings erschwerend hinzukommt, ist die Tatsache, dass keine der drei Aufgaben losgelöst von der anderen $\mathrm{zu}$ betrachten ist und nur die Beantwortung aller drei Fragekomplexe zu einem Fortschritt in der Sache führen kann. Auch wenn die Klärung des Literalitätsbegriffes gelingen sollte, ist für die Liminalitätsforschung nicht viel gewonnen, und das Gleiche gilt natürlich für den umgekehrten 
Fall. Die Frage nach dem Zusammenhang von Literalität und Liminalität geht über diese schon von sich aus sicherlich nicht gering einzuschätzenden Schwierigkeiten insofern noch einmal hinaus, als erst von der scheinbar unverbindlichen Kopula »und« genaue Rückschlüsse auf die Richtung zu erwarten sind, die das Forschungsprojekt nehmen wird. Die Klärung der Begriffe Literalität und Liminalität dient daher vor allem der Vorbereitung der zielführenden Bestimmung des Verhältnisses von Literalität und Liminalität, wobei sich dem »Ideal der letzten Erfüllung«, das Edmund Husserl in den Logischen Untersuchungen als Übereinstimmung von Bedeutungsintention und Bedeutungserfüllung gekennzeichnet hat, jederzeit das Schreckgespenst eines »Ideals der letzten Enttäuschung « ${ }^{1}$ beigesellt, falls sich die Intentionen, die mit dem Projekt verbunden sind, eben nicht erfüllen lassen.

Vor dem Hintergrund dieser komplexen Ausgangssituation ist die Aufgabe der folgenden Ausführungen also zunächst einmal relativ klar konturiert: In einem ersten Schritt geht es um die begriffliche Klärung der Ausdrücke Literalität und Liminalität, um auf dieser Grundlage mögliche Anschlusspunkte zwischen beiden Bereichen zu markieren, ohne in die doppelte Gefahr einer völligen Unverbindlichkeit oder einer zu großen begrifflichen Enge zu fallen. Das Ziel der folgenden Überlegungen besteht demzufolge darin, von der Frage, was eigentlich gemeint ist, wenn von Literalität und Liminalität gesprochen wird, bis zu der weiterführenden Frage vorzudringen, was in Zukunft geleistet werden kann, wenn sich gezeigt hat, dass die Begriffe Literalität und Liminalität nicht nur für sich unverbunden nebeneinanderstehen, sondern sich wechselseitig ergänzen und so unterschiedlichen Forschungsprojekten Raum geben.

\section{»Die Schrift ist unveränderlich«. Zum Begriff der Literalität}

»Richtiges Auffassen einer Sache und Missverstehen der gleichen Sache schließen einander nicht vollständig aus «, ${ }^{2}$ sagt der Gefängniskaplan in Kafkas Proce $\beta$ zu K., als es um die Frage nach der richtigen Auslegung der Schrift geht, von der der Kaplan behauptet, sie sei zwar unveränderlich, zugleich aber »ein Ausdruck der Verzweiflung darüber « ${ }^{3}$. Nichts

1 Vgl. Achim Geisenhanslüke: »Das Ideal der letzten Enttäuschung. Dekonstruktivistische Literaturwissenschaft«, in: Komparatistik. Jahrbuch der Deutschen Gesellschaft für Allgemeine und Vergleichende Literaturwissenschaft (2004/2005), S. 77-89.

2 Franz Kafka: Der Proceß, Frankfurt a.M. 1994, S. 229.

3 Ebd., S. 230. 
scheint in und aufgrund seiner Unveränderlichkeit unsicherer zu sein als die Schrift, und die Verzweiflung, in ihrer eigentlichen Form nach Kierkegaard der Versuch, »verzweifelt man selbst sein wollen «, ${ }^{4}$ betrifft jede Möglichkeit, sich über die Schrift klar werden zu wollen. So ist auch der Begriff der Schrift in Kafkas Text in seiner scheinbaren Unveränderlichkeit zugleich vielfältig: als Heilige Schrift, mosaische Gesetzesschrift oder als verborgener Gesetzeskodex. Jede dieser Schriftformen hat zugleich unterschiedliche Deutungsversuche vorgegeben, die an Kafkas Werk herangetragen worden sind. Für jede der Schriftformen, die im Proceß wirksam zu sein scheinen, gilt aber auch, dass sie durch die $» k l e i n e n$ Winkelzüge $\ll^{5}$, als die Kafka das eigene Schreiben bezeichnet hat, gekonnt unterlaufen werden. Wenn Schrift bei Kafka zum Thema wird, dann also in mehrfacher Hinsicht: in der Konkurrenz unterschiedlicher theologischer Modelle, die einen je unterschiedlichen Umgang mit der Schrift pflegen wie das Christentum und das Judentum, im Gegensatz von profaner und religiöser Schrift, zwischen Recht und Offenbarung, in der hermeneutischen Vieldeutigkeit der Auslegung, die die Schrift erfahren kann, und schließlich in der scheinbar naiven Frage nach Kafkas persönlicher $>$ Handschrift $<$ als Schriftsteller.

Die Vieldeutigkeit, die die Bestimmung der Schrift bei Kafka betrifft, kann sicherlich nicht einfach auf das Problem der Schrift überhaupt übertragen werden. Sie kann aber als Hinweis auf die Problemart dienen, mit der es das Denken der Schrift zu tun hat. Die Schwierigkeit besteht in der unterschiedlichen Zielrichtung der vorliegenden Ansätze, Schrift zu denken. Nicht jeder Ansatz, der sich mit Schrift beschäftigt, will das gleiche Phänomen erklären. So geht es den Untersuchungen zum Verhältnis von Oralität und Literalität, wie sie Jack Goody und Eric A. Havelock vorgelegt haben, um etwas grundsätzlich anderes als die Dekonstruktion der abendländischen Metaphysik am Leitfaden der Unterscheidung von Stimme und Schrift, die Jacques Derrida unternommen hat, oder Nelson Goodmans »Ansatz zu einer allgemeinen Symboltheorie « 6 , die auf der Grundlage der Exemplifikation als »wichtige und vielverwendete Weise der Symbolisierung innerhalb und außerhalb der Künste $\ll^{7}$ auf den Zusammenhang von Literatur und Inskription abzielt, demzufolge »Texte keine Partituren, sondern Skripten ${ }^{8}$ sind. Auch wenn die Literalitätsforschung, die Dekonstruktion und Nelson Goodmans kogni-

4 Søren Kierkegaard: Die Krankheit zum Tode, Düsseldorf 1954, S. 8.

5 Franz Kafka: Briefe an Felice, Frankfurt a.M. 1976, S. 83.

6 Nelson Goodman: Sprachen der Kunst. Entwurf einer Symboltheorie, Frankfurt a.M. 1988, S. 9.

7 Ebd., S. 59.

8 Ebd., S. 159. 
tive Theorie ästhetischer Erfahrung über den gleichen Begriff - eben Schrift - zu sprechen scheinen: Die unterschiedliche Zielrichtung ihrer Ansätze lässt eine zufriedenstellende Vermittlung kaum zu. ${ }^{9}$ So sympathisch die Idee gerade im Kontext liminalen Denkens auch zunächst erscheinen mag: Es kann nicht darum gehen, Brücken zwischen unterschiedlichen Ansätzen zu bauen, die unterschiedliche Sachverhalte erklären möchten. Vielmehr besteht die vorbereitende Aufgabe in der genauen Differenzierung der verschiedenen Ansätze, um abschließend abwägen zu können, wo sich Berührungspunkte in der Sache ergeben.

\section{Auf der Schwelle: Zum Begriff der Liminalität}

Nicht nur zum Begriff der Schrift, auch zum Problem der Liminalität gibt Kafka im Proceß-Roman aufschlussreiche Hinweise. Die These des Kaplans über die Unveränderlichkeit der Schrift folgt unmittelbar auf die berühmte Parabel Vor dem Gesetz, in der der Mann vom Lande Einlass in das Gesetz begehrt. Mit dem Hinweis, der Eintritt sei möglich, jetzt aber nicht, wird ihm der Wunsch verwehrt, bis die Pforte des Gesetzes mit dem Tod des Mannes und dem zweiten Hinweis geschlossen wird, dass nur er hier hätte Einlass erlangen können.

So rätselhaft Kafkas Parabel, die immer wieder die unterschiedlichsten Deutungen erfahren hat, ${ }^{10}$ auch anmuten mag: Kafkas Mann vom Lande befindet sich im Sinne der ethnologischen Forschung in einer klassischen Schwellensituation, innerhalb eines einmal begonnenen und dann unterbrochenen Übergangsritus, der erst mit dem Tod abgeschlossen wird. Wie Arnold van Gennep in seiner Untersuchung zu den rites de passage gezeigt hat, zeichnen sich Übergangsriten durch drei miteinander verknüpfte Phasen aus: »Übergangsriten erfolgen also, theoretisch zumindest, in drei Schritten: Trennungsriten kennzeichnen die Ablösungsphase, Schwellen- bzw. Umwandlungsriten die Zwischenphase (die Schwellen- bzw. Umwandlungsphase) und Angliederungsriten die Integ-

9 Vgl. dazu die Ausführungen von Florian Coulmas: »Die Anliegen der einzelnen Disziplinen im Zusammenhang mit der Schrift sind verschieden, die Probleme teilweise ähnlich. Sie sind jedoch so vielfältig, dass sie in keiner Disziplin erschöpfend behandelt werden können. Eine Grammatologie gibt es nicht und wird es nicht geben.« Florian Coulmas: Über Schrift, Frankfurt a.M. 1981, S. 11.

10 Exemplarisch bei Klaus-Michael Bogdal (Hg.): Neue Literaturtheorien in der Praxis. Textanalysen von Kafkas >Vor dem Gesetz‘, Opladen 1993, und Oliver Jahraus/Stefan Neuhaus (Hg.): Kafkas >Urteil und die Literaturtheorie. Zehn Modellanalysen, Stuttgart 2002. 
rationsphase. ${ }^{11}$ Die liminale Phase markiert demnach eine Zwischenposition. Ihr voraus geht eine Situation der Krise, die die Trennung von einer bisher etablierten Ordnung betrifft, wobei auf die Krise die Wiedereingliederung in die alte Struktur folgt. Die Schwellenphase dient demnach vor allem dazu, mögliche schädliche Auswirkungen auf die soziale Gemeinschaft durch ein genau abgestuftes Ritual zu verhindern.

Um die rites de passage näher zu beschreiben, geht van Gennep von einem Zusammenhang zwischen Liminalität und Räumlichkeit aus. $»$ Räumliche Übergänge « ${ }^{12}$ so van Gennep, bilden das Modell der von ihm geschilderten Übergänge. Die Räume können dabei in unterschiedlichen Formen figurieren, als Tor, Bodenschwelle, Oberschwelle oder Tragbalken. Wichtig ist allein die Übergangssituation: »Jeder, der sich von einer Sphäre in die andere begibt, befindet sich eine Zeitlang sowohl räumlich als auch magisch-religiös in einer besonderen Situation: er schwebt zwischen zwei Welten. « $^{13}$

Kafkas Parabel schildert eine Situation, die van Genneps Theorie der Übergangsriten in vielerlei Hinsicht vergleichbar ist. Zunächst ist auch die Erfahrung des Mannes vom Lande durch eine bestimmte Form der Räumlichkeit geprägt: Er sucht Eingang in einen sakral konnotierten Innenraum, von dem er sich einiges für sein Seelenheil zu versprechen scheint, kommt aber über die Schwelle nicht hinaus. Der Türhüter, dessen Funktion zugleich an eine allegorische Darstellung psychoanalytischer Erklärungsmuster denken lässt, markiert die Instanz des Verbotes, den Zensor, der den Mann nicht hineinlässt und damit einen Schwellenzustand herstellt, der sich von dem von van Gennep herausgearbeiteten Modellfall insofern unterscheidet, als zunächst keine Phase der Reintegration mehr erfolgt, die Schwellensituation in der Form des Wartens also zu einer ständigen wird. Darüber hinaus verkörpert der Protagonist von Kafkas Roman einen Grenzgänger in genau dem Sinne, in dem van Gennep annimmt, dieser schwebe zwischen zwei Welten: der alltäglichen, durch berufliche Zwänge und diverse mehr oder minder zufällige Frauenbekanntschaften strukturierten Welt sowie der Welt des Gesetzes, die ihn wie den Homo sacer Giorgio Agambens mit einer Art Bann belegt hat, der zu seiner abschließenden Hinrichtung führt. Auch in diesem Fall zeigt sich, dass der Protagonist des Romans durch die Verhaftung, mit der der Proce $\beta$ einsetzt, in eine permanente Schwellensituation versetzt wird. Der Beginn des Romans verweist schließlich noch auf eine dritte Weise auf das Thema der Schwelle. Der Text beginnt wie auch etwa Die

11 Arnold van Gennep: Übergangsriten (Les rites de passage), Frankfurt a.M., New York 2005, S. 21.

12 Ebd., S. 25.

13 Ebd., S. 27. 
Verwandlung mit dem Moment des Erwachens, das allerdings ebenso gut ein Einschlafen und Eintauchen in einen Traum sein könnte. »Wir sind sehr arm an Schwellenerfahrungen geworden. Das Einschlafen ist vielleicht die einzige, die uns geblieben ist $\ll,{ }^{14}$ notiert Walter Benjamin im Blick auf Proust und Kafka zu der marginalisierten Rolle von Schwellenerfahrungen in der Moderne. Wie kaum ein anderer Schriftsteller seiner Zeit scheint Kafka nun die Schwelle von Schlaf, Traum und Erwachen zu thematisieren, die Walter Benjamin und Sigmund Freud auf unterschiedliche Art und Weise zum Thema ihrer historischen Untersuchungen genommen haben. ${ }^{15}$

Vor diesem Hintergrund hat Winfried Menninghaus den Begriff der »Schwellenkunde« geltend gemacht, um Benjamins Passage des Mythos zu beschreiben. Menninghaus kann die Idee einer Schwellenkunde auf das Werk Benjamins beziehen, da dieser einer der Ersten ist, der scharf zwischen Schwelle und Grenze unterscheidet: »Die Schwelle ist ganz scharf von der Grenze zu scheiden. Schwelle ist eine Zone. Wandel, Übergang, Fluten liegen im Worte `schwellen` und diese Bedeutung hat die Etymologie nicht zu übersehen. Andererseits ist es notwendig, den unmittelbaren tektonischen und zeremonialen Zusammenhang festzustellen, der das Wort zu seiner Bedeutung gebracht. $"{ }^{16}$ Wie insbesondere das Passagen-Werk zeigt, stellt das Denken Walter Benjamins einen der prominentesten Versuche dar, den Begriff der Schwelle in das Zentrum der historischen Erkenntnis zu stellen.

Die Schwierigkeiten, die mit dem Begriff der Liminalität in diesem Kontext verbunden sind, sind allerdings andere als im Fall der Literalität. Dem Begriff der Schwelle, auf den sich das Denken der Liminalität beruft, kommt ein metaphorischer Charakter zu, der eine begriffliche Erklärung zunächst eher zu erschweren als zu ermöglichen scheint. ${ }^{17}$ So hat schon Rolf Parr darauf hingewiesen, „dass die Rede von Grenzen, Schwellen und Passagen fast immer symbolischen oder metaphorischen

14 Walter Benjamin: Das Passagen-Werk. Erster Band, Frankfurt a.M. 1982, S. 617.

15 Vgl. Susan Buck-Morss: Dialektik des Sehens. Walter Benjamin und das Passagenwerk, Frankfurt a.M. 1993, S. 556f.

16 W. Benjamin: Das Passagen-Werk, S. 618.

17 Schon die Arbeitsgruppe um Nicholas Saul hat den Begriff der Schwelle vor allem als Metapher, ja als »Metapher der Metapher« verstanden, ohne allerdings den theoretischen Voraussetzungen nachzugehen, die das metaphorische Sprechen von der Schwelle impliziert. Vgl. Nicholas Saul/Daniel Möbus/Birgit Illner (Hg.): Schwellen. Germanistische Erkundungen einer Metapher, Würzburg 1999, S. 10. 
Charakter hat «. ${ }^{18}$ Die Frage, die sich im Kontext der Liminalitätsforschung stellt, ist die nach dem Erkenntniswert, der der Metapher der Schwelle zugewiesen werden kann. Der metaphorische Gehalt, der dem Begriff der Liminalität zukommt, verbindet sich darüber hinaus wie schon im Fall der Literalität mit heterogenen Ansätzen, die sich auf ganz unterschiedliche Weise mit dem Begriff auseinandergesetzt haben. Wie Rolf Parr gezeigt hat, stammen wichtige Anregungen zum Thema der Liminalität neben der Ethnologie und dem Werk Benjamins aus so unterschiedlichen Richtungen wie der Ritualtheorie Victor Turners, Genettes Analyse der Paratexte, der Foucault'schen Diskursanalyse oder Giorgio Agambens Projekt des Homo sacer. Gerade die metaphorische Qualität des Schwellenbegriffs führt zu einer Unübersichtlichkeit, die leicht in Beliebigkeit umschlagen kann. Folgt man an dieser Stelle Benjamins Begriff der Schwelle, der, wie Menninghaus hervorgehoben hat, »in einem dreifachen Sinn: in seiner geschichtsphilosophischen Intention, seiner wissenschaftlichen Form und seinem Hauptgegenstand « ${ }^{19}$ Passagen markiert, so ergeben sich dennoch Gemeinsamkeiten zwischen den unterschiedlichen Schwellenbegriffen. Wie sich bereits bei Kafka gezeigt hat, geht es der Liminalität zunächst um spezifisch räumlich strukturierte Formen des Übergangs. In der gleichen Weise spielt aber eine zeitliche Dimension herein: Zum einen sind die Schwellen, von denen Benjamin spricht, in ein geschichtsphilosophisches Modell eingebettet, dem es um Phänomene der Erinnerung und des Vergessens geht. Die Ritualforschung beschreibt liminale Situationen ebenfalls im Hinblick auf zeitliche Ordnungen, wenn sie bestimmte Krisenzeiten wie Adoleszenz, Hochzeit, Tod aus sozialanthropologischer Perspektive zum Gegenstand nimmt. Schließlich ist die Frage nach der Liminalität mit Fragen nach sozialer Gemeinschaft verbunden. Am deutlichsten wird das bei Victor Turner, der von der Voraussetzung ausgeht, »dass die Entscheidung, ein Ritual auszuführen, sehr oft mit Krisen im Sozialleben der Dorfbewohner zusammenhing «. ${ }^{20}$ Turner etabliert daher einen engen Zusammenhang von Liminalität und Communitas, zwischen Schwellenerfahrungen und Fragen der sozialen Ordnung, wobei er der Liminalität »das Zwischen-

18 Rolf Parr: Liminale und andere Übergänge. Theoretische Modellierungen von Grenzzonen, Normalitätsspektren, Schwellen, Übergängen und die Zwischenräume in Literatur- und Kulturwissenschaft, S. 16 des vorliegenden Bandes. Rolf Parr sei an dieser Stelle für seine vielfältigen Anregungen zum Thema der Liminalität gedankt.

19 Winfried Menninghaus: Schwellenkunde. Walter Benjamins Passage des Mythos, Frankfurt a.M. 1986, S. 49f.

20 Victor Turner: Das Ritual. Struktur und Anti-Struktur, Frankfurt a.M. 2005, S. 17. 
stadium der Statuslosigkeit $\ll^{21}$ zuspricht - jener Statuslosigkeit, die den Protagonisten von Kafkas Romanen auf so ausgezeichnete Art und Weise zukommt. Dass das Phänomen der Liminalität etwas mit literarischen Formen zu tun hat, deutet Turner selbst an, wenn er feststellt:

Kann man den Schwellenzustand der Riten der Lebenskrisen vielleicht ein wenig kühn mit der Tragödie vergleichen - denn beide beinhalten Demütigung, Entblößung und Schmerz -, so kann man den Schwellenzustand der Statusumkehrung vielleicht mit der Komödie vergleichen, da beide mit spöttischer Nachahmung und Inversion, nicht aber mit der Vernichtung der Strukturrollen und ihrer übereifrigen Anhänger einhergehen. ${ }^{22}$

Die Aufgaben der literaturwissenschaftlichen Liminalitätsforschung liegen dementsprechend darin, das Potential, das Theoretiker wie Benjamin oder Turner freigesetzt haben, zu nutzen, zum Beispiel bei der Frage nach der Überlagerung von Tragödie und Komödie und dem Einbruch karnevalesken Lachens im Werk Kafkas. Vor diesem Hintergrund erscheint es sinnvoll, auf der Grundlage der bisher skizzierten Konstellation vor allem auf die eingangs unterschiedenen Felder der Literalitätsforschung und der Dekonstruktion näher einzugehen, um von dort aus Möglichkeiten der Überschneidung mit dem Thema der Liminalität neu zu überdenken.

\section{Das Medium der Schrift}

In seinen Überlegungen zum Tod des Autors in der modernen Literatur vertritt Roland Barthes einleitend die These, „que l'écriture est destruction de toute voix, de toute origine «. ${ }^{23}$ Wie auch immer Barthes' Begriff der écriture im Kontext einer strukturalen oder poststrukturalen Poetik genauer zu fassen ist: Mit den anthropologischen und soziologischen Literalitätsstudien, die Autoren wie Jack Goody, Eric A. Havelock und Walter J. Ong vorgelegt haben, scheint er kaum etwas gemein zu haben. Während Barthes mit dem Begriff der écriture eine dezidiert avantgardistische Theorie und Praxis des literarischen Schreibens anvisiert, geht es der Sozialanthropologie um den historischen Übergang von oralen zu literalen Gesellschaften und damit um die Veränderungen, die die Schrift für die unterschiedlichen sozialen Systeme mit sich brachte. In seiner all-

21 Ebd., S. 97.

22 Ebd., S. 191.

23 Roland Barthes: »La mort de l'auteur«, in: ders.: Le bruissement de la langue. Essais critiques IV, Paris 1984, S. 63-69. 
gemeinen Bedeutung umfasst der Begriff der Literalität demzufolge alle sprachlichen und kulturellen Erscheinungsformen, die durch die Schrift in die Gesellschaft gekommen sind. Als Gegenbegriff zur Oralität meint Literalität eine Manuskript- und Inschriftenkultur, die auf der Speicherung und Weitergabe von kulturellen Inhalten in textlich fixierten Formen basiert.

Geht es der Literalitätsforschung demnach zunächst um die Aufarbeitung eines historischen Sachverhalts, um die Anfänge der Schriftkultur und die Schwelle zwischen oralen und literalen Gesellschaften, so überschneidet sich ihr Erkenntnisinteresse in diesem Punkt mit dem der Medienwissenschaft. Wenn es der Schrift vor allem um Speicherung und Weitergabe von Wissen geht, dann ist das Phänomen der Literalität Bestandteil einer übergreifenden Mediengeschichte, die durch die Stationen der Handschrift, des Buchdrucks und der elektronischen Speicherung gekennzeichnet ist: »Die Schrift, der Druck, die Computertechnologie - das sind Meilensteine der Technologisierungsgeschichte des Wortes «, ${ }^{24}$ hält Walter J. Ong fest. Im Rahmen einer Mediengeschichte der Literalität erscheint das elektronische Zeitalter im Blick auf Telefon, Radio und Fernsehen im Unterschied zur primären Oralität, die die Personen betrifft, die Schreiben nicht kennen, zugleich als Zeit einer >sekundären Oralität , die sich mit den neuen Medien durchgesetzt hat.

Dabei haben sich unterschiedliche Themenkreise herauskristallisiert, die das Interesse der Literalitätsforschung bestimmen. Geht es ihr zunächst um den Übergang von oralen zu literalen Kulturen, so besteht in der Forschung ein breiter Konsens darüber, dass Literalität Oralität auf gewisse Weise voraussetzt, gesprochene Rede also historisch vor Prozessen der Verschriftlichung steht. Das zeigt sich besonders an der griechischen Kultur, die mit der Einführung des Alphabets die Grundlagen der modernen literalen Kulturen geschaffen hat. Wie Havelock deutlich gemacht hat, ist die griechische Literatur und Philosophie von »wachsender Spannung zwischen den Formen mündlicher und dokumentierter Rede $\ll^{25}$ gekennzeichnet. Havelock kommt im Laufe seiner Untersuchung zur Schriftlichkeit zu überraschenden Thesen, die ihren Ausgang von der Einsicht in die Präliteralität der Griechen nehmen. So stellen Homers Dichtungen nach Havelock »keine Literatur im modernen Sinne dar, sondern oral gespeicherte Erfahrung ${ }^{26}{ }^{26}$, und auch die Vorsokratiker, insbesondere Xenophanes, Heraklit und Parmenides, erscheinen in seinen

24 Walter J. Ong: Oralität und Literalität. Die Technologisierung des Wortes, Opladen 1987, S. 83.

25 Eric A. Havelock: Schriftlichkeit. Das griechische Alphabet als kulturelle Revolution, Weinheim 1990, S. 80.

26 Ebd., S. 116. 
Augen als Reaktionen auf eine soziale Umgebung, in der sich die Literalität noch nicht voll durchgesetzt hatte. Havelock, der Milman Parrys Arbeiten zum Zusammenhang von Oralität und Epos bei Homer aufnehmen kann, wendet sich damit gegen die drohende Herabsetzung der Oralität und kommt zu dem Schluss, dass eine »Auffassung, die kulturelle Verfeinerung mit einem gewissen Grad von Literalität gleichgesetzt [sic!], abgelehnt werden $\ll{ }^{27}$ muss.

Ein zweiter Schwerpunkt der Literalitätsforschung liegt im Thema der Funktion der Schrift als Träger der Erinnerung beschlossen. So stellt Jack Goody in seiner Untersuchung zur Literalität in traditionalen Gesellschaften fest, Sprache könne durch Schrift »über räumliche Entfernungen übermittelt und durch die Zeit hindurch bewahrt werden; was Menschen sagen und denken, kann vor der Vergänglichkeit mündlicher Kommunikation gerettet werden ${ }^{28}{ }^{28}$ Goody geht in seinen Überlegungen zur Literalität auf die traditionelle Bestimmung der Schrift als Medium der Erinnerung ein, die schon Platon im Phaidros entwickelt hat. Soziale Kulturen scheinen damit wesentlich von Schrift abhängig zu sein. Steht im Mittelpunkt der Prähistorie der Mensch als biologisches Wesen, so betrachten Historie, Anthropologie und Soziologie den Menschen als erinnerndes Wesen, indem sie Literalität als Grund sozialer Organisation sichtbar machen.

Was von sozialer Bedeutung bleibt, wird im Gedächtnis gespeichert, während das übrige in der Regel vergessen wird: und Sprache - in erster Linie das Vokabular - ist das wirksame Medium dieses wichtigen Prozesses sozialer Verdauung und Ausscheidung, die man als ein Analogon zur homöostatischen Organisation des menschlichen Körpers, vermittels deren er sein Dasein zu erhalten sucht, auffassen kann. ${ }^{29}$

Erinnerung erscheint als ein wesentlich sozialer Prozess, Sprache als wichtigstes Instrument zur Unterscheidung dessen, was erinnert oder vergessen werden soll. So konnte der Zusammenhang zwischen Schriftlichkeit und der Funktion des kulturellen Gedächtnisses, den Halbwachs und Goody etabliert haben, auch von Jan Assmann zur Grundlage der kulturwissenschaftlichen Bestimmung der Erinnerung gemacht werden. ${ }^{30}$ Assmann spricht in diesem Zusammenhang vom Übergang von ritueller

27 Ebd., S. 41.

28 Jack Goody: »Einleitung«, in: ders. (Hg.): Literalität in traditionalen Gesellschaften, Frankfurt a.M. 1981, S. 7-43, hier S. 8.

29 Ebd., S. 50.

30 Vgl. Jan Assmann: Das kulturelle Gedächtnis. Schrift, Erinnerung und politische Identität in frühen Hochkulturen, München 1992. 
zu textueller Kohärenz als dem Übergang von Wiederholung zu Vergegenwärtigung, von Nachahmung zu Auslegung, um Veränderungen in der Geschichte zu markieren, die auf der einen Seite die Aufgabe einer Archäologie des literarischen Textes notwendig machen, auf der anderen Seite eine kritische Bestimmung der hermeneutischen Auslegungstechnik fordern.

Damit rückt jedoch zugleich ein Problem ins Blickfeld, das dazu zwingt, den Boden der historisch-anthropologischen Literalitätsforschung zu verlassen: die Frage nach der theoretischen Begründung des Schriftbegriffs, die Assmann wie selbstverständlich im den Bereich der Hermeneutik findet. Hatte schon Friedrich Schleiermacher die Hermeneutik als »die Kunst, die Rede eines andern, vornehmlich die schriftliche, richtig zu verstehen ${ }^{31}$, definiert, so spricht er mit Rede und Schrift die Unterscheidung von Mündlichkeit und Schriftlichkeit an, ohne jedoch deren Implikationen weiter zu beachten. Damit stellt sich die Frage, ob die von Assman anvisierte Archäologie des literarischen Textes nicht verstärkt auf andere theoretische Zugänge reagieren müsste. Gerade die Dekonstruktion, die wie keine andere philosophische Theorie ihrer Zeit den Begriff der Schrift ins Zentrum ihrer Überlegungen gestellt hat, bietet sich in diesem Kontext an, um die kulturhistorischen Implikationen von Literalitätsforschung und Gedächtnistheorie kritisch zu hinterfragen.

\section{Die Schrift der Dekonstruktion}

Den Ausgangspunkt der Literalitätsforschung bildet die Frage nach dem Verhältnis von Oralität und Literalität aus der Perspektive von Soziologie, Anthropologie und Geschichtswissenschaft. Einen ganz anderen $\mathrm{Zu}$ gang zum Thema der Schrift gewinnt Jacques Derridas Dekonstruktion der abendländischen Geschichte der Metaphysik. Derrida verfolgt unter dem Titel Grammatologie eine Theorie, die zum einen die Semiologie Saussures und zum anderen die Seinsphilosophie Heideggers auf neue Grundlagen zu stellen versucht, indem sie nicht mehr vom sprachlichen Zeichen oder dem Sein spricht, sondern von der Schrift.

Derridas eigene Ambitionen scheinen dabei keineswegs darin zu liegen, eine neue Theorie oder Methode zu begründen. Vielmehr betont er einleitend, dass »nous n'ayons pas l'ambition d'illustrer une nouvelle méthode $\ll$, dass es ihm vielmehr um »des problèmes de lecture critique ${ }^{32}$ gehe. Die kritische Lektüre, die Derrida in der Grammatologie

31 Friedrich Schleiermacher: Hermeneutik und Kritik, Frankfurt a.M. 1977, S. 71.

32 Jacques Derrida: De la grammatologie, Paris 1967, S. 7. 
ankündigt, richtet sich vor allem auf »la question du texte, de son statut historique, de son temps et de son espace propre $«{ }^{33}$ Nicht historischempirische Veränderungen nimmt Derrida in den Blick, wenn er von Schrift spricht, sondern - das hat die Dekonstruktion gerade für die Literaturwissenschaft interessant werden lassen - Fragen des Textes. Anders als etwa bei Roland Barthes, der einen ähnlich emphatisch besetzten Begriff der écriture vertritt, geht es Derrida jedoch nicht vorrangig um die Frage nach literarischer Textualität. Im Mittelpunkt seiner Überlegungen steht vielmehr die Bedeutung der Konzepte Schrift und Text für die Philosophie und die Geschichte der Metaphysik.

Die Ausgangsthese, die Derrida im Anschluss an Heidegger in der Grammatologie entwickelt, betrifft die Geschichte der Metaphysik als die von Anwesenheit und Wahrheit. Derrida zufolge geht es vor allem darum, dass »1'histoire de la vérité, de la vérité de la vérité, a toujours été, à la différence près d'une diversion métaphorique dont il nous faudra rendre compte, l'abaissement de l'écriture et son refoulement hors de la parole $>$ pleine « $\ll{ }^{34}$ Schon am Beispiel der Phänomenologie Husserls hatte Derrida in Die Stimme und das Phänomen erläutert, dass die Privilegierung der Stimme vor der Schrift den Ursprungsakt metaphysischer Philosophie bedeute, den es im Rahmen seiner kritischen Lektüre zu hinterfragen gelte. ${ }^{35}$ Auf den ersten Blick vollzieht Derrida daher so etwas wie eine Umwertung der traditionellen philosophischen Bestimmung des Verhältnisses von Schrift und Stimme, wenn er behauptet: »A tous les sens de ce mot, l'écriture comprendrait le langage. $«{ }^{36}$ Besteht die Stimme in der zeichentheoretischen Begrifflichkeit des Strukturalismus in der lautlichen Äußerung einer Bedeutung, im Signifikanten, der ein Signifikat hervorruft, so wäre die Schrift als bloße Verdoppelung des Signifikanten für Derrida als Signifikant des Signifikanten zu bestimmen. Da Sprache aber insgesamt auf einer unendlichen Kette von Signifikanten beruht, wie Derrida im Blick auf Saussure und die Erweiterung des linguistischen Zeichenmodells durch Lacan konstatiert, stellt Schrift das System der Sprache überhaupt dar: »Le signifié y fonctionne toujours déjà comme un signifiant «, ${ }^{37}$ lautet die Auflösung des traditionellen Verhältnisses von Signifikant und Signifikat, die mit dem Spiel der unendlich aufeinander verweisenden Signifikanten zugleich das traditionelle Verhältnis von Stimme und Schrift aus den Angeln hebt. Schrift ist nicht länger Derivat der Stimme, sondern ihr verborgener Grund.

33 Ebd., S. 8.

34 Ebd., S. 12.

35 Vgl. Jacques Derrida: La voix et le phénomène, Paris 1967.

36 J. Derrida: De la grammatologie, S. 16.

37 Ebd. 
Die These, Schrift sei in ihrer traditionellen philosophischen Bestimmung als Signifikant eines Signifikanten zu fassen, verbindet Derridas Kritik der Metaphysik mit einer zweiten Idee, die vor allem der Philosophie Heideggers entlehnt ist. Sie zielt auf »la détermination historiale du sens de l'être en générale comme présence « ${ }^{38}$ So wie Heidegger der Geschichte der Metaphysik unterstellt, das Sein immer als eine Form der Gegenwart, als Präsenz zu denken, so geht Derrida von der Prämisse aus, die Stimme habe in der Geschichte immer für eine Form der Präsenz gestanden, die Schrift hingegen für die Absenz der Sinnerfüllung der Sprache in der Stimme. Heideggers Geschichte der Seinsvergessenheit wird Derrida zur Geschichte der Schriftvergessenheit, die die Metaphysik seit ihren Ursprüngen in der griechischen Antike kennzeichne. Und so wie Heidegger das Ereignis eines neuen Denkens des Seins zu ermöglichen sucht, so situiert Derrida seinen eigenen Diskurs am Ende einer Epoche, die durch Schriftvergessenheit gekennzeichnet ist.

Derridas Strategie besteht daher vordergründig in einer Aufhebung des traditionellen Verhältnisses von Oralität und Literalität. Entscheidend ist in diesem Zusammenhang jedoch, dass der Begriff der Schrift von der Dekonstruktion nicht unberührt bleibt. Derridas Ausgangsthese lautet, dass »le signe linguistique implique une écriture originaire « ${ }^{39}$ Die ursprüngliche Form der Schrift, von der Derrida ausgeht, deckt sich jedoch weder mit dem Konzept der Oralität noch mit dem der Literalität: »Nous pensons au contraire que la langue orale appartient déjà à cette écriture. Mais cela suppose une modification du concept d'écriture que nous ne faisons pour l'instant qu'anticiper. ${ }^{40}{ }^{4}$ Die Umwertung des Verhältnisses von Oralität und Literalität führt daher zu einem neuen und anderen Schriftbegriff. »Il ne s'agit donc pas ici de réhabiliter l'écriture au sens étroit, ni de renverser l'ordre de dépendance lorsqu'il est évident $«{ }^{41}$ stellt Derrida fest, um zu unterstreichen, »que le langage >originel<, snaturek etc., n'ait jamais existé, qu'il n'ait jamais été intact, intouché par l'écriture, qu'il ait toujours été lui-même une écriture. Archi-écriture dont nous voulons ici indiquer la necessité et dessiner le nouveau concept «. ${ }^{42}$ Wenn Derrida Sprache und Schrift bis zur Ununterscheidbarkeit einander annähert, dann geschieht das nicht im Blick auf den traditionellen Schriftbegriff, wie ihn auch die Literalitätsforschung kennt. Hatte diese stets vorausgesetzt, dass die Einführung der Literalität die Oralität voraussetzt, so geht Derrida einen umgekehrten Weg: Jede Form der

38 Ebd., S. 23.

39 Ebd., S. 77.

40 Ebd., S. 81.

41 Ebd., S. 82.

42 Ebd., S. 82f. 
Sprache, so die Prämisse, setze die Schrift voraus, eine Form der Schrift allerdings, die sich dem traditionellen Verständnis von Schrift entzieht und die Derrida daher mit dem Begriff der »archi-écriture« einführt, um sie als ein Prinzip zu kennzeichnen, das jeder Unterscheidung von Oralität und Literalität vorausgeht:

La trace (pure) est la difference. Elle ne depend d'aucune plenitude sensible, audible ou visible, phonique ou graphique. Elle en est au contraire la condition. Bien qu'elle n'existe pas, bien qu'elle ne soit jamais un étant-présent hors de toute plénitude, sa possibilité est antérieure en droit à tout ce qu'on appelle signe (signifié/signifiant, contenu/expression etc.), concept ou operation, motrice ou sensible. Cette difference n'est donc pas plus sensible qu'intelligible et elle permet l'articulation des signes entre aux à l'intérieur d'un même ordre abstrait - d'un texte phonique ou graphique par exemple - ou entre deux orders d'expression. Elle permet l'articulation de la parole et de l'écriture - au sens courant - comme elle fonde l'opposition métaphysique entre le sensible et l'intelligible, puis entre signifiant et signifié, expression et contenu etc. ${ }^{43}$

Derrida kennzeichnet seinen Begriff der archi-écriture in Anlehnung an Freud und Heidegger als Spur oder différance. Damit meint er allerdings kein Ursprungskonzept, aus dem heraus die Gegensätzlichkeit von Stimme und Schrift entspringt. Wenn Derrida die Differenz als Spur kennzeichnet, dann zielt er auf die Dekonstruktion der Gegensätze durch ein ihnen vorausliegendes Prinzip, das als solches nie aufweisbar ist, ohne dass die metaphysischen Gegensätze aber auch nicht zu denken sind. Die Urschrift als Spur oder Differenz ist daher weder mit dem empirischen Begriff der Schrift identisch noch mit einem metaphysischen Begriff der Urschrift. Als Ursprung aller sprachlichen Differenzen und Bedeutungen ist die Schrift als Spur zugleich die Abwesenheit eines Ursprungs in der Form einer mythischen Präsenz, die am Anfang der Unterschiede stünde: »La trace est en effet l'origine absolue du sens en général. Ce qui revient à dire, encore une fois, qu'il n'y a pas d'origine absolue du sens en general. La trace est la differance qui ouvre l'apparaître et la signification. " $^{44}$ Die Spur als différance deutet Derrida nicht als Prinzip zeitlicher Gegenwärtigkeit, sondern als eine Bewegung, die in nichts anderem als der Tätigkeit des ständigen Differierens besteht:

Ce qui s'écrit difference, ce sera donc le movement de jeu qui sproduits, par ce qui n'est pas simplement une activité, ces differences, ces effets de differences. Cela ne veut pas dire que la difference qui produit les differences, soit avant elles, dans un present simple et en soi immodifié, in-différent. La difference est

43 Ebd., S. 92.

44 Ebd., S. 95. 
l'>origine〈 non-pleine, non-simple, l'origine structuré et différante des differences. Le nom d'>origine ne lui convient donc plus. ${ }^{45}$

Derrida geht es demzufolge nicht etwa um das Herausarbeiten einer Archi-Struktur der Sprache, sondern um eine Position, aus der heraus sich die Vergangenheit - in diesem Fall die Geschichte der Metaphysik als beständige Folge der Supplementierungen des Ursprungs ergibt, die die différance zu maskieren versuchen. Derridas Überlegungen zur Schrift stehen in der Tradition der Versuche zur Überwindung der Metaphysik, wie sie Nietzsche und Heidegger vorgelegt haben, so dass ein Brückenschlag zu empirisch und historisch ausgerichteten Formen der Schriftforschung fast unmöglich zu sein scheint.

Gerade aus der Perspektive der Literalitätsforschung und ihrer Erweiterung durch die Medienwissenschaft hat Derridas Theorie daher einige Kritik erfahren müssen. Ein gängiger Vorwurf lautet, er habe »das Abhängigkeitsverhältnis der Schrift von der Sprache geradezu in sein Gegenteil verkehrt und einen zu engen Schriftbegriff eintauscht gegen einen viel zu weiten $\ll .{ }^{46}$ Die Kritik richtet sich auf die scheinbare Überbietung des Gegensatzes von Oralität und Literalität durch den Begriff der Urschrift. Den Vorwurf, Derrida tausche einen zu engen gegen einen zu weiten Schriftbegriff, hat Albrecht Koschorke aus medientheoretischer Perspektive aufgenommen und weiter ausgeführt. Auch Koschorke konstatiert, Derrida »weitet den Begriff der écriture über die Materialität des Mediums hinaus in Richtung auf eine allgemeine artikulatorische Struktur aus, die jeden Signifikationsakt, auch das Sprechen, erfasst $\ll .{ }^{47}$ Koschorkes eigenes Interesse liegt daher darin, die Dekonstruktion in einer allgemeinen »Mediologie« aufzuheben, die deren Intentionen besser realisiere.

Koschorkes Leitthese zur Kritik Derridas lautet, dass die dekonstruktive These der Schriftfeindschaft der Metaphysik »aus einem seinerseits schriftkulturell bedingten Phantasma ${ }^{48}{ }^{4}$ hervorgeht. Um seine These $\mathrm{zu}$

45 Jacques Derrida: Marges de la philosophie, Paris 1972, S. 12.

46 Gernot Grube/Werner Kogge/Sybille Krämer (Hg.): Schrift. Kulturtechnik zwischen Auge, Hand und Maschine, München 2005, S. 11. Zur Kritik an Derrida vgl. auch Christoph Türcke: Vom Kainszeichen zum genetischen Code. Kritische Theorie der Schrift, München 2005, S. 170-190. Türckes ambitioniertes Projekt erscheint in mancherlei Hinsicht jedoch als von der Frankfurter Schule inspirierter Rückfall hinter die »neue« Kritik, die durch Autoren wie Derrida und Foucault repräsentiert wird.

47 Albrecht Koschorke: Körperströme und Schriftverkehr. Mediologie des 18. Jahrhunderts, München 2003, S. 327.

48 Ebd., S. 328. 
belegen, geht Koschorke auf Derridas Platonkritik zurück. Gegen die Auffassung der Schriftlichkeit, die die Dekonstruktion der Metaphysik unterstellt, macht Koschorke - in diesem Punkt eher in Übereinstimmung mit den Forschungsergebnissen von Havelock - geltend, dass Platons Kritik an der Schrift selbst auf einer Logik des Literalen beruht: »Alles spricht dafür, dass die Idee der entkörperten Stimme [...] ein Schrifteffekt ist. ${ }^{49}$ Koschorke wirft Derrida daher vor, durch die strikte Entgegensetzung von Schriftlichkeit und Mündlichkeit eine Selbstpräsenz der Stimme vorauszusetzen, die er eigentlich dekonstruieren möchte. Die unmittelbare Folge aus dieser Kritik besteht in der Überführung der Dekonstruktion in eine Mediologie:

Eine Mediologie nach diesem Verständnis hat nicht das Problem, den Nachweis zu führen, dass alle metaphysischen Identitäten sich selbst durchkreuzen, weil es ihnen niemals gelingt, den semiotischen Verweisraum unter Kontrolle zu halten, aus dem sie begrifflich hervorgehen. Sie ist nicht vorrangig daran interessiert, Ursprungs-, Ganzheits-, Einheits- und Wahrheitsansprüche immanent philosophisch, wenn auch durch Abschreitung der logischen Ränder der Philosophie, in Zweifel zu ziehen. Ihr ist vielmehr an der Frage gelegen, wie solche Phantasmata [...] positiv funktionieren und sich die Macht eines sozialen und technischen Realitätsprinzips aneignen können. Differentialität erscheint dann als ein Teilmoment auf dem Feld der durchaus gelingenden und kontrollierenden Zeichenverwendung, und das Erkenntnisziel besteht darin, ihre Systemstelle konkret $\mathrm{zu}$ bestimmen. ${ }^{50}$

Koschorke vollzieht damit eine Überwindung der Dekonstruktion, die für sich in Anspruch nimmt, die Ziele derselben besser realisieren zu können:

Es stellt sich also die Frage, ob die Dekonstruktion über ein hinreichendes Instrumentarium verfügt, um die funktionalen Leistungen der Schrift im besonderen und von den Medien im allgemeinen erkennbar zu machen; oder ob man sie nicht gleichsam aus einer Vierteldrehung über sich hinaustreiben und aus ihrem Beharren im Bann einer negierten Metaphysik herauslösen muss, um zu einem genuin >mediologischen` Verständnis von Wirklichkeitskonstruktionen durch Zeichen zu kommen. ${ }^{51}$

Die Pirouette, die Koschorke mit der und über die Dekonstruktion hinaus dreht, um auf »eine unaufgeregte Weise $\aleph^{52} \mathrm{zu}$ ähnlichen Ergebnissen zu gelangen, schraubt sich in eine neue Höhe der Theorie hinauf. Ihr Name

49 Ebd., S. 339.

50 Ebd., S. 345.

51 Ebd.

52 Ebd. 
ist nicht mehr Dekonstruktion, sondern Mediologie, ihr Ahnvater nicht mehr Derrida, sondern Luhmann. So luzide Koschorkes Kritik der Dekonstruktion aber auch erscheinen mag: Ihr Vorwurf, die Dekonstruktion setzte die Prämisse eines illiteraten Logos nur durch einen Akt der UrInskription, ist keine Kritik, sondern eine Bestätigung Derridas. Nichts anderes hatte dieser ja in der Grammatologie behauptet, wo er die archiécriture als jeder Unterscheidung von Oralität und Literalität vorgängige Instanz dargestellt hat. Die kritische Frage, der sich Koschorke stellen muss, ist daher die, ob die Frage nach dem positiven Funktionieren nicht hinter die »kritische Lektüre« zurückfällt, die Derrida propagiert hatte. Berechtigt ist sicherlich der Hinweis auf Derridas Zugehörigkeit zu einem spezifisch philosophischen Diskurs, die dieser allerdings auch nie geleugnet hat. $\mathrm{Zu}$ fragen wäre aber, ob und inwiefern das kritische Potential, das die Dekonstruktion der Philosophie in der Tradition von Nietzsche und Heidegger entfaltet, nicht auch auf Fragen des literarischen Diskurses auszuweiten wäre. Gerade Derridas eigene Überlegungen zum Problem der Schwelle im Werk Kafkas und Celans etwa führen in eine Richtung, die sich mit dem Themenfeld der Liminalität zunächst zu decken scheint. Die Frage, inwiefern aus dekonstruktiver Perspektive gerade die Literatur ein geeignetes Objekt für die Verknüpfung von Literalität und Liminalität wäre, scheint daher einer der möglichen Ansatzpunkte des Forschungsprojektes zu sein.

\section{Schriftkultur und Schwellenkunde}

Die Auseinandersetzung mit den unterschiedlichen Schriftbegriffen aus den Bereichen der Literalitätsforschung und der Dekonstruktion hat zunächst bestätigt, dass in beiden Fällen völlig unterschiedliche Schriftbegriffe vorliegen, die nicht einfach in Übereinstimmung zu bringen sind. Während Ethnologie und Sozialanthropologie das Verhältnis von Oralität und Literalität in einem geschichtlich-empirischen Sinne als Ausgangspunkt dient, demzufolge Literalität Oralität jederzeit voraussetzt, entwickelt die Dekonstruktion einen Schriftbegriff, der für sich in Anspruch nimmt, den Gegensatz von Mündlichkeit und Schriftlichkeit außer Kraft zu setzen, indem er ihn im Konzept der archi-écriture geradezu auf den Kopf stellt. Die Frage, die sich auf der Grundlage der derart miteinander unvereinbaren Schriftbegriffe stellt, ist die, ob und wie sie sich auf sinnvolle Weise mit dem Begriff der Liminalität in Verbindung bringen lassen.

Die grundsätzliche Schwierigkeit, die dem Begriff der Liminalität im Unterschied zu dem der Literalität anhaftet, besteht in dem eingangs 
erörterten Problem, dass der Rede von Grenzen und Schwellen ein eher metaphorischer Charakter zukommt, der auf alles andere als auf begriffliche Eindeutigkeit zielt. Dennoch lässt sich auch im Falle der Liminalität eine relativ klare Differenzierung unterschiedlicher Ansätze und Zielrichtungen ausmachen. Rolf Parr unterscheidet in diesem Zusammenhang zwischen vier unterschiedlichen Modellen, die das Denken der Liminalität prägen:

[e]rstens ein einfaches, eine dyadische Unterscheidung schaffendes Modell ohne Übergangszone als Grundmodell von Grenze [...], zweitens eine Abwandlung des Grundmodells zu einer mit wie auch immer fungierenden Übergangszone [...], drittens ein triadisches Modell mit Übergangszone und teleologischer Prozessialität [...] und viertens ein triadisches Modell mit zwei Übergangszonen und permanenter, nicht konsequent auf ein Ziel hinauslaufender Prozessualität. ${ }^{53}$

Das erste Modell zielt auf den Begriff der Grenze als klarer Trennungslinie ab, während die anderen Modelle auf unterschiedliche Art und Weise den Begriff der Schwelle anvisieren. Dabei entspricht das dritte Modell mehr oder weniger dem ethnologischen Liminalitätsdenken im Sinne van Genneps und Turners, das vierte Modell hingegen eher einem dekonstruktiven oder diskursanalytischen Ansatz, dem es darum geht, sowohl den scharfen Begriff der Grenze als auch den teleologisch ausgerichteten Schwellenbegriff Turners in eine Form der permanenten Krisenerfahrung zu überführen, auf die Derridas Erschütterung der Geschichte der Metaphysik ebenso abzielt wie Foucaults frühe, dem Werk Batailles abgelesene Theorie der Überschreitung. ${ }^{54}$

Die Frage nach den möglichen Verschränkungsbereichen von Literalität und Liminalität kann demzufolge zunächst bei den Überschneidungen ansetzen, die sich aus dem Zusammenhang zwischen dem dekonstruktiven Schriftbegriff und dem Modell von Liminalität als Zone permanenter Prozessualität ergibt. Angesichts der grundlegenden Metaphorizität des Schwellenbegriffs sollte dabei allerdings deutlich geworden sein, dass es hier nicht um den Aufbau eines logischen Gerüsts geht, das in eine einheitliche Theorie führen könnte. Vielmehr handelt es sich gerade bei der Frage nach der Verschränkung von Literalität und Liminalität um die Vermessung eines Terrains, das ständig in Bewegung ist und sich logischen Grenzziehungen verweigert. Wenn deutlich geworden ist, dass den unterschiedlichen Schriftbegriffen der Literalitäts- wie den me-

53 R. Parr: Liminale und andere Übergänge, S. 47f.

54 Vgl. Michel Foucault: »Vorrede zur Überschreitung«, in: Schriften zur Literatur, Frankfurt a.M. 2003, S. 64-85. 
taphorischen Erklärungsmodellen der Liminalitätsforschung vor allem ein heuristischer Wert zukommt, dann lassen sich unterschiedliche Schnittfelder markieren, die das Interesse des Forschungsprojekts bestimmen können. Auf einer ersten Ebene, die zunächst nicht viel mehr als eine Analogie zwischen dekonstruktiven, diskursanalytischen und literarischen Verfahren zu verkörpern scheint, in ihrer Bedeutung jedoch nicht zu unterschätzen ist, rücken literarische und außerliterarische Texte in den Blick, in denen das Thema der Schwelle als Gegenstand wie als Konstituent der eigenen Form eine zentrale Rolle spielt, wie es zum Beispiel bei Franz Kafka oder bei Paul Celan der Fall ist. In diesem Zusammenhang stellt sich zugleich eine weitere grundsätzliche Frage, die den Kern des Forschungsprojektes betrifft: die nach den Gemeinsamkeiten und Unterschieden zwischen den Begriffen der Literalität und der Literarizität, dem Verhältnis von materieller Schrift und der Idee einer poetischen Funktion der Sprache, wie sie schon der Strukturalismus entworfen hat, sowie dem Phänomen der Buchstäblichkeit als Grundlage und Grenze des Verstehens von Texten.

Damit rückt zugleich ein zweites Problem des Forschungsprojektes in den Blick, die Frage nach der geschichtlichen Bestimmung von Literatur im Blick auf den Zusammenhang von Prozessen der Verschriftlichung und Schwellenerfahrungen. Eine besondere Bedeutung kommt in diesem Zusammenhang der Ablösung starrer epochaler Zäsuren, die das literaturgeschichtliche Denken bis heute geprägt hat, durch eine Auffassung zu, die im Anschluss an Koselleck, Foucault und andere Geschichte als einen Prozess begreift, der sich durch zum Teil langdauernde Schwellenzeiten auszeichnet, die jede eindeutige Periodisierung fragwürdig machen. ${ }^{55}$ Erscheint Literatur im Kontext der Geschichte als eine besondere Form der schriftlichen Überlieferung, die nicht in allen Kulturen gleichermaßen zu finden ist - Walter J. Ong betont, dass Sprache ein vorherrschend orales Phänomen sei und dass von den vielen tausend Sprachen »nur ungefähr 78 eine Literatur $~^{56}$ hervorgebracht haben -, so sind literarische Texte immer auch als transitorische Phänomene zu begreifen, die keine festen Anfangs- oder Endpunkte kennen, sondern Übergänge verkörpern, die selbst wiederum als Schwellen fungieren.

Vor diesem Hintergrund kann es nicht verwundern, dass die Einführung der neuen Medien in die Geschichte von einigen Theoretikern zugleich als Ende der Schrift gedeutet wurde. Das gilt nicht nur für die Me-

55 Vgl. Reinhart Herzog/Reinhart Koselleck (Hg.): Epochenschwelle und Epochenbewußtsein, München 1987, sowie Hans-Ulrich Gumbrecht/Ursula Link-Heer (Hg.): Epochenschwellen und Epochenstrukturen im Diskurs der Literatur- und Sprachhistorie, Frankfurt a.M. 1985.

W. J. Ong: Oralität und Literalität, S. 15. 
dientheorie Marshall McLuhans, der das Ende der Gutenberggalaxis in Aussicht stellt, sondern auch für Vilém Flusser:

Schreiben im Sinne einer Aneinanderreihung von Buchstaben und anderen Schriftzeichen scheint kaum oder überhaupt keine Zukunft zu heben. Es gibt mittlerweile Codes, die besser als die der Schriftsteller Informationen übermitteln. Was bisher geschrieben wurde, kann besser auf Tonbänder, Schallplatten, Filme, Videobänder, Bildplatten oder Disketten übertragen werden. ${ }^{57}$

Das Ende der Schrift bedeute daher auch das Ende der Literatur:

Der Buchdruck, dieses selbstbewusst gewordene alphabetische Schreiben, kann als der selbstbewusst gewordene Ausdruck des westlichen, geschichtlichen, wissenschaftlichen, fortschrittlichen Denkens angesehen werden. Die informatische Revolution macht den Buchdruck, das Alphabet und dieses Denken überflüssig. ${ }^{58}$

Flusser sieht die Kulturgeschichte nach der Literalität vor einer erneuten Zäsur, die mit dem Privileg der Schrift aufräumt. Schrift, das Medium, dem es selbst wesentlich um Speicherung historischer Daten geht, scheint dem Vergessen überantwortet. Wie auch immer Flussers wohlfeiler Abgesang auf das Zeitalter der Literalität zu beurteilen ist: Die informatische Revolution lässt sich selbst nicht als Ende, sondern als eine neue Kultur der Inskription beschreiben. Vor diesem Hintergrund öffnet sich im Schnittfeld von Literalität und Liminalität ein dritter, medientheoretisch und -historisch besetzter Raum. Scheint die Frage nach Medien schon von sich aus auf grundsätzliche Art und Weise nach einer Position des »Zwischen« zu zielen, da Medien selbst nichts anderes als transitorische Zwischenräume verkörpern, so wäre zum einen die grundsätzliche Funktion der Medien als fundamentale Zwischenräume in kulturellen Prozessen zu klären, zum anderen die Frage nach dem Verhältnis von Literatur und Formen sekundärer Oralität zu stellen, wie sie sich nicht nur im Blick auf Telefon, Radio und Fernsehen ergibt, sondern auch in Texten etwa von Louis-Ferdinand Céline, Rainhard Jirgl, Thomas Kling und anderen Autoren, die Oralität in der Schrift inszenieren, um sie zu dekonstruieren.

Eine vierte Bedeutung, die der Zusammenhang von liminalen und literalen Aspekten annehmen kann, liegt in der kulturwissenschaftlichen Frage nach Grenzregionen als Räumen, die von Schwellen und Über-

57 Vilém Flusser: Die Schrift. Hat Schreiben Zukunft?, Frankfurt a.M. 1992, S. 7.

58 Ebd., S. 50. 
schreitungen geprägt sind. ${ }^{59}$ In einem zunächst ganz vortheoretischen Sinn sind Grenzräume mit der Inszenierung kultureller Praktiken und Kontakte verbunden, die zu eigenen literarischen und medialen Transformationen führen. Das gilt in besonderer Weise für Räume, die von Phänomenen der Mehrsprachigkeit und der kulturellen Hybridität geprägt sind wie das Saarland, Luxemburg, aber auch Regensburg, das sich in seinem kulturellen Selbstverständnis als Brücke nach Südosteuropa zu erkennen gibt.

Die Frage nach Formen kultureller Hybridität führt schließlich noch zu einer fünften Bedeutung, die der Zusammenhang von Literalität und Liminalität annehmen kann. Aus der Perspektive der gender-Forschung lassen sich kulturelle Schwellen zugleich zur Infragestellung scheinbar eindeutiger Geschlechtergrenzen nutzen, wie sie etwa in der Figur des Hermaphroditen vorliegt, dessen scheinbar monströse Existenz in philosophische, literarische und medizinische Diskurse auf gleichermaßen beunruhigende Weise eingegangen ist. ${ }^{60}$ Der Verschriftlichung und Aufhebung von Geschlechtergrenzen wie dem begleitenden Thema der Monstrosität in literarischen und außerliterarischen Texten nachzugehen, erscheint als eine weitere lohnenswerte Perspektive des Forschungsprojektes.

Damit erhebt sich sicherlich ein Einwand, der die einleitende Frage nach dem Kern des Forschungsprojektes und dem Zusammenhang von Literalität und Liminalität wieder aufnimmt. Sicher stünde es einem Projekt, das sich in wesentlichen Teilen auf den Begriff der Schwelle stützt, schlecht an, allzu eindeutige Grenzziehungen vornehmen zu wollen. Die Heterogenität der hier vorgestellten Ansätze wie möglicher Arbeitsfelder aber scheint zunächst den Eindruck der Unbestimmtheit zu bestätigen, der mit dem Projekt verbunden ist. Einen Ausweg aus diesem Dilemma zu finden, der dem Zusammenhang von Schrift, Literatur und Schwelle ein theoretisches Fundament geben könnte, wäre jedoch der falsche Weg. Die bisher noch ungenutzten Möglichkeiten der Literalitäts- und Liminalitätsforschung liegen vielmehr gerade in der mit ihrer begrifflichen Unbestimmtheit einhergehenden Breite, die es ermöglicht, literaturtheoretische, medienhistorische und kulturwissenschaftliche Ansätze unter einem Dach zu versammeln. Es wird daher eher von den konkreten Gegenständen als von einem theoretischen Konzept abhängen, welche Richtung ein Projekt nehmen wird, das nach dem Schriftcharakter literarischer Texte

59 Vgl. Rüdiger Görner: Grenzen, Schwellen, Übergänge. Zur Poetik des Transitorischen, Göttingen 2001, sowie Dieter Lamping: Über Grenzen. Eine literarische Topographie, Göttingen 2001.

60 Vgl. Michel Foucault: Über Hermaphrodismus. Der Fall Barbin, Frankfurt a.M. 1998. 
als Träger und Speicher kulturellen Wissens wie nach der literarischen Inszenierung der Schwelle als einem permanenten Zustand der Überschreitung gleichermaßen fragt, um Grenzen in ihrer vielfältigen Bedeutung nicht zu stabilisieren, sondern in einer kritischen Lektüre außer Kraft zu setzen.

\section{Literatur}

Assmann, Jan: Das kulturelle Gedächtnis. Schrift, Erinnerung und politische Identität in frühen Hochkulturen, München 1992.

Barthes, Roland: »La mort de l'auteur«, in: ders.: Le bruissement de la langue. Essais critique IV, Paris 1984, S. 63-69.

Benjamin, Walter: Das Passagen-Werk. Erster Band, hg. v. Rolf Tiedemann, Frankfurt a.M. 1982.

Bogdal, Klaus-Michael (Hg.): Neue Literaturtheorien in der Praxis. Textanalysen von Kafkas >Vor dem Gesetz‘, Opladen 1993.

Buck-Morss, Susan: Dialektik des Sehens. Walter Benjamin und das Passagen-Werk, Frankfurt a.M. 1993.

Coulmas, Florian: Über Schrift, Frankfurt a.M. 1981.

Derrida, Jacques: La voix et le phénomène, Paris 1967.

Derrida, Jacques: De la grammatologie, Paris 1967.

Derrida, Jacques: Marges de la philosophie, Paris 1972.

Flusser, Vilém: Die Schrift. Hat Schreiben Zukunft?, Frankfurt a.M. 1992.

Foucault, Michel: Über Hermaphrodismus. Der Fall Barbin, Frankfurt a.M. 1998.

Foucault, Michel: »Vorrede zur Überschreitung«, in: ders.: Schriften zur Literatur, Frankfurt a.M. 2003, S. 64-85.

Geisenhanslüke, Achim: »Das Ideal der letzten Enttäuschung. Dekonstruktivistische Literaturwissenschaft «. Komparatistik. Jahrbuch der Deutschen Gesellschaft für Allgemeine und Vergleichende Literaturwissenschaft (2004/2005), S. 77-89.

Gennep, Arnold van: Übergangsriten (Les rites de passage), Frankfurt a.M., New York 2005.

Goodman, Nelson: Sprachen der Kunst. Entwurf einer Symboltheorie, Frankfurt a.M. 1988.

Goody, Jack: »Einleitung«, in: ders. (Hg.): Literalität in traditionalen Gesellschaften, Frankfurt a.M. 1981, S. 7-43.

Görner, Rüdiger: Grenzen, Schwellen, Übergänge. Zur Poetik des Transitorischen, Göttingen 2001. 
Grube, Gernot/Kogge, Werner/Krämer, Sybille (Hg.): Schrift. Kulturtechnik zwischen Auge, Hand und Maschine, München 2005.

Gumbrecht, Hans-Ulrich/Link-Herr, Ursula (Hg.): Epochenschwellen und Epochenstrukturen im Diskurs der Literatur- und Sprachhistorie, Frankfurt a.M. 1987.

Havelock, Eric A.: Schriftlichkeit. Das griechische Alphabet als kulturelle Revolution, Weinheim 1990.

Herzog, Reinhart/Koselleck, Reinhart (Hg.): Epochenschwelle und Epochenbewußtsein, München 1987.

Jahrhaus, Oliver/Neuhaus, Stefan (Hg.): >Kafkas Urteilı und die Literaturtheorie. Zehn Modellanalysen, Stuttgart 2002.

Kafka, Franz: Briefe an Felice, Frankfurt a.M. 1976.

Kafka, Franz: Der Proceß, Frankfurt a.M. 1994.

Kierkegaard, Søren: Die Krankheit zum Tode, Düsseldorf 1954.

Koschorke, Albrecht: Körperströme und Schriftverkehr. Mediologie des 18. Jahrhunderts, München 2004.

Lamping, Dieter: Über Grenzen. Eine literarische Topographie, Göttingen 2001.

Menninghaus, Winfried: Schwellenkunde. Walter Benjamins Passage des Mythos, Frankfurt a.M. 1986.

Ong, Walter J.: Oralität und Literalität. Die Technologisierung des Wortes, Opladen 1987.

Parr, Rolf: Liminale und andere Übergänge. Theoretische Modellierungen von Grenzzonen, Normalitätsspektren, Schwellen, Übergängen und die Zwischenräume in Literatur- und Kulturwissenschaft, im vorliegenden Band S. 11-63.

Saul, Nicholas/Möbus, Daniel/Illner, Birgit (Hg): Schwellen. Germanistische Erkundungen einer Metapher, Würzburg 1999.

Schleiermacher, Friedrich: Hermeneutik und Kritik, Frankfurt a.M. 1977. Türcke, Christoph: Vom Kainszeichen zum genetischen Code. Kritische Theorie der Schrift, München 2005.

Turner, Victor: Das Ritual. Struktur und Anti-Struktur, Frankfurt a.M. 2005. 
